\title{
CORRECTION
}

\section{Correction to: Effect of Polyvinyl Chloride Microplastics on Bacterial Community and Nutrient Status in Two Agricultural Soils}

\author{
Yuanyuan Yan ${ }^{1} \cdot$ Zhanghao Chen $^{1} \cdot$ Fengxiao Zhu ${ }^{1} \cdot$ Changyin Zhu ${ }^{1} \cdot$ Chao Wang $^{1} \cdot$ Cheng Gu $^{1}$
}

Published online: 19 August 2020

(c) Springer Science+Business Media, LLC, part of Springer Nature 2020

\section{Correction to: \\ Bulletin of Environmental Contamination and Toxicology https://doi.org/10.1007/s00128-020-02900-2}

The original version of the article unfortunately contained a mistake in Fig. 3. In this figure, norank_o_DS-100 and Niastella were mistakenly labelled with $\alpha$-Proteobacteria and Firmicutes, respectively. The correct lineage is given below.

Norank_o_DS-100 is within the phylum Acidobacteria, while Niastella belongs to the phylum Bacterioidetes.

This has been corrected with this erratum.

Publisher's Note Springer Nature remains neutral with regard to jurisdictional claims in published maps and institutional affiliations.

The original article can be found online at https://doi.org/10.1007/ s00128-020-02900-2.

Fengxiao Zhu

fengxiao@nju.edu.cn

1 State Key Laboratory of Pollution Control and Resource Reuse, School of the Environment, Nanjing University, Nanjing 210023, People's Republic of China 